Check for updates

Cite this: RSC Adv., 2017, 7, 49102

Received 29th August 2017

Accepted 14th October 2017

DOI: $10.1039 / \mathrm{c} 7 \mathrm{ra09594e}$

rsc.li/rsc-advances

\section{Ultra-high resolution in low field tabletop NMR spectrometers $\uparrow$}

\author{
Kavitha Rachineni, \$๋ Veera Mohana Rao Kakitał and Ramakrishna V. Hosur (D)*ab
}

\begin{abstract}
At the present time, when NMR spectrometers are getting prohibitively expensive, for routine applications in chemistry where NMR is an indispensable technique, working with tabletop low field spectrometers would be an attractive proposition. However, as is well known, chemical shift dispersion is a serious limitation on these systems. The modern strategies of pure shift spectroscopy would not be useful because of lack of gradients, on the one hand, and even when they are there, there will be an extreme loss of sensitivity by slice selective gradients in the pulse sequences, on the other. The present manuscript presents an approach for resolution enhancement by employing processing-based advancements in the pure shift NMR schemes and presents data acquired on a $60 \mathrm{MHz}$ NMR spectrometer. We use generalized indirect covariance $(\mathrm{GIC})$ processing based reconstruction for enhancing the resolution in 2D-NMR spectra and that has been demonstrated on different small organic molecule samples.
\end{abstract}

Development of analytical methods for structural chemistry is very important in understanding the modifications that have taken place during chemical reactions. In this respect, spectroscopic techniques have been well recognized to obtain accurate information on analytes (either it could be a pure molecule or mixture of small molecules). Amongst them, NMR has been proven to provide the atomic level structural information of chemical constituents present in the reaction processes. ${ }^{1}$ The advent of high-field super conducting magnets with cryo-probe technologies has made the chemical structural analysis simple. Laterally, the development of cryogenic free permanent field homogeneous tabletop magnets has made a significant impact as this is cost effective, a lot less maintenance and does not require great NMR expertise to operate them. Indeed, several analytical applications of tabletop NMR systems have been reported (edible oils, ${ }^{2}$ fats, ${ }^{3}$ quality control of pharmaceutical drugs, ${ }^{4}$ food,${ }^{5}$ mobile sensor-MRI ${ }^{6}$ and reaction monitoring $\left.{ }^{7-10}\right)$. Further, because of the low costs, these instruments have found a common place in undergraduate educational laboratories.

It is well known that 2D-NMR experiments significantly enhance spectral resolution and facilitate unambiguous analysis. However, at low magnetic fields, severely overlapped scalar coupling multiplets belonging to different chemical sites hamper the chemical shift resolution, even in 2D-NMR spectra

\footnotetext{
${ }^{a} U M-D A E$ Centre for Excellence in Basic Sciences, University of Mumbai, Kalina Campus, Santacruz, Mumbai 400 098, India.E-mail: hosur@tifr.res.in

${ }^{b}$ Department of Chemical Sciences, Tata Institute of Fundamental Research (TIFR), 1Homi Bhabha Road, Colaba, Mumbai 400 005, India

$\dagger$ Electronic supplementary information (ESI) available: Complete experimental and step by step processing details are given. See DOI: 10.1039/c7ra09594e

\$ These authors contributed equally.
}

and this need to be sorted out to be able to identify the molecules present in a reaction mixture. In this sense, pure shift NMR methods help to enhance the resolution by obtaining exclusively chemical shift information (couplings are removed) in the spectra. ${ }^{11}$ On the other hand, the various sophisticated pure shift NMR methods such as, versions of Zangger-Sterk, ${ }^{12}$ BIRD,${ }^{13}$ HOBS $^{14}$ and PSYCHE $^{15}$ broadband decoupling methods, that have been developed on modern high field spectrometers, are difficult to implement on low field spectrometers and are currently not available.

The present manuscript offers a solution to the above discussed resolution problems in low field 2D-NMR spectra by invoking the ideas of recently proposed processing based pure shift NMR i.e.; GIC (generalized indirect covariance) method. ${ }^{16-18}$ Indeed the importance of covariance processing has been explored for different homodecoupling methods..$^{19} \mathrm{~A}$ pre-requisite to conduct GIC is a pure shift one dimensional NMR spectrum. We build such a spectrum from the homodecoupled trace of $2 \mathrm{D}$ $\mathrm{J}$-res ${ }^{20}$ spectrum. This is then used to build a 2D diagonal spectrum (see ESI $\dagger$ for the full details) which is then used to process all other 2D spectra to obtain their pure shift versions (processing has been done on MNOVA 11). The versatility of the present GIC based 2D pure shift NMR has been demonstrated for ibuprofen, trans-2-pentenal and a mixture of trans-2-pentenal and propanol, on a $60 \mathrm{MHz}$ Oxford tabletop NMR spectrometer.

Fig. 1 shows a comparison of conventional COSY and GICCOSY of ibuprofen, a standard drug, recorded on a $60 \mathrm{MHz}$ spectrometer. Clearly, the conventional COSY spectrum has severely overlapped multiplets, especially in methyl and methylene regions (Fig. 1a), whereas, in the GIC pure shift COSY (Fig. 1b) all the correlations belonging to different chemical sites are well resolved. 


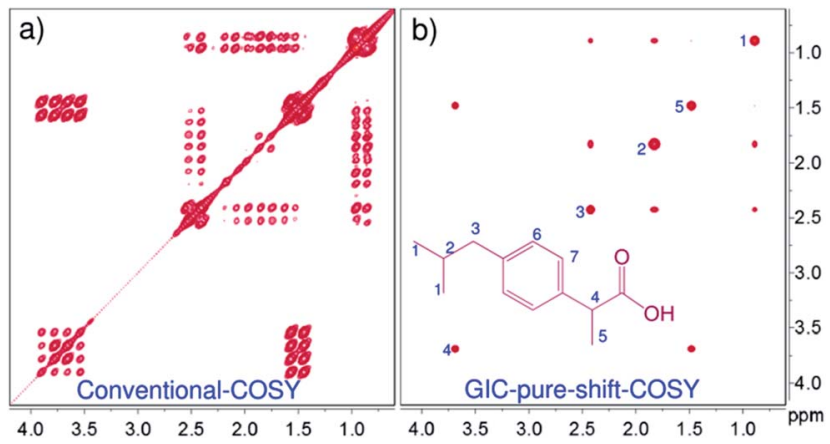

Fig. 1 Comparison of expanded methyl and methylene chemical sites of ibuprofen: the conventional COSY (a) resonances with scalar coupling multiplets, and the GIC based pure shift COSY with homodecoupled resonance contours (b).

Fig. 2 compares the resolution enhancement in the processing based pure shift TOCSY spectrum over the conventional-2D spectrum of trans-2-pentenal. Clearly, the resolution in the GIC pure shift TOCSY spectrum is far superior and all the correlations can be unambiguously established. Fig. 3 shows the applicability of such techniques even for a mixture of two molecules and the individual correlations can be clearly established using GIC pure shift COSY and GIC pure shift TOCSY spectra.

Finally, a word has to be mentioned about the limitations of the present method, i.e.: appearance of some covariance artefacts for the very closely separated multiplets. For example, the GIC pure shift COSY spectrum of the present mixture has shown processing based artefacts between the $\mathrm{H} 5$ and $\mathrm{H} 6$ protons, although they belong to two independent molecules. Similar artefacts are even seen in the TOCSY as well, i.e., between the H5 and $\mathrm{H} 7$ protons. Therefore, a little care should be taken while analyzing the GIC generated pure shift NMR data, as we have compared herein with the original 2D NMR spectra. Otherwise, pure shift GIC spectra have to be generated at different values of matrix powers. ${ }^{17}$ Additionally, quality of the pure shift GIC spectra mainly depends on the homodecoupled trace that we obtain from the J-res experiments. At low magnetic field

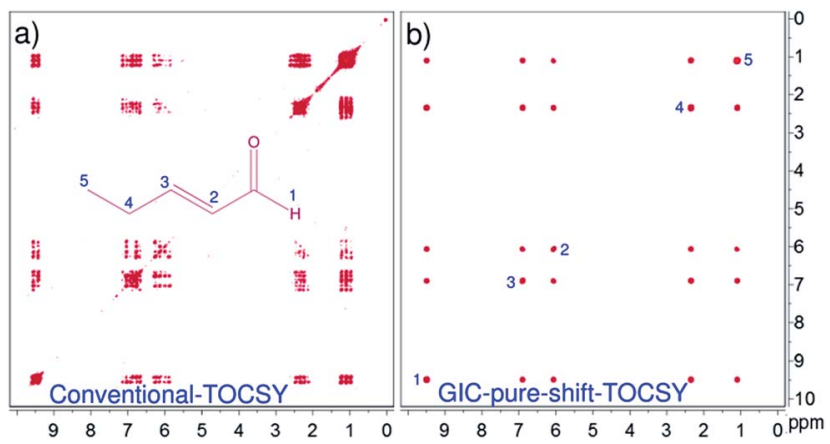

Fig. 2 Comparison of 2D-homonuclear spectra recorded for trans-2pentenal, before and after the GIC based formalism. Herein, chemical shift resonances in the conventional-TOCSY (a) with the overlapped multiplets are nicely resolved in the GIC based pure shift TOCSY (b).

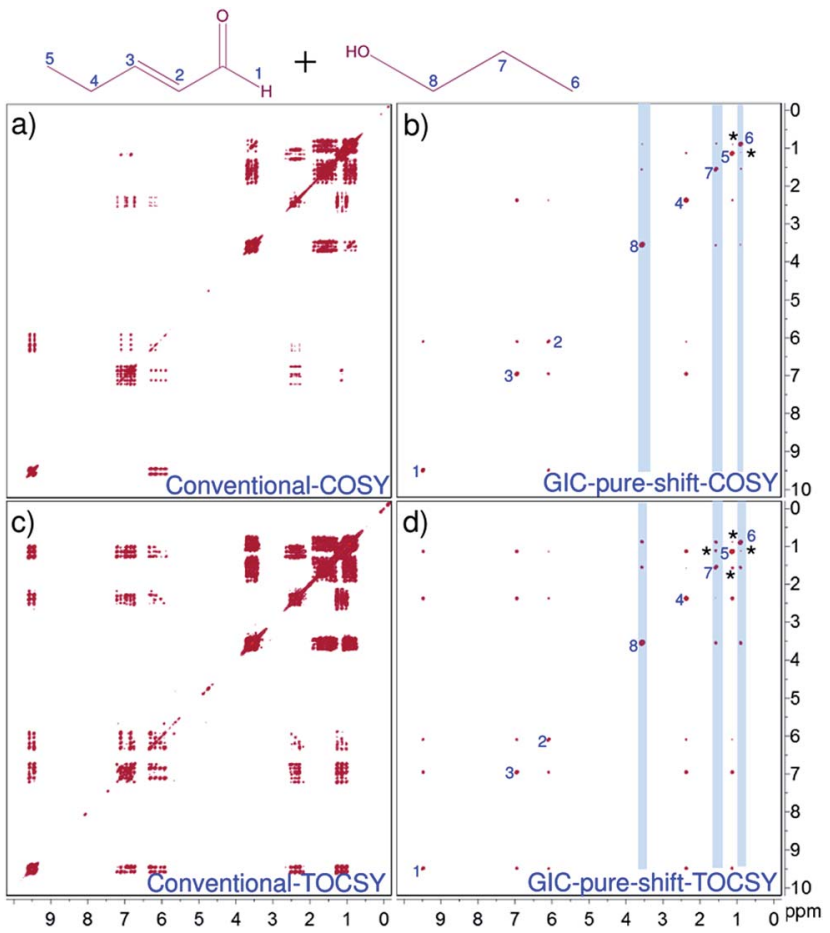

Fig. 3 Illustrating the importance of GIC based processing in the analysis of small organic molecule mixture i.e.; a hypothetical mixture of trans-2-pentenal and propanol, where two molecules have been differentiated by highlighting propanol resonances with the light blue colour strips. Herein, comparison of the conventional COSY (a) and TOCSY (c) with the corresponding GIC based pure shift COSY (b) and TOCSY (d) spectra has made understanding the significance of resolution enhancement (GIC processing) in analyzing the complex organic mixtures. The cross-peaks which are depicted with the asterisk (*) symbols represent the covariance artefacts.

strengths, there is a possibility of having strong coupling information; in such situations obtaining clean pure shift trace is frequently hampered. This problem can be circumvented by simulating the pure shift trace from the real homodecoupled Jres projection (as we did in the present studies), while omitting the strong coupling artefacts. As shown here, this strategy has worked well even for only $\sim 12 \mathrm{~Hz}$ separation (protons $\mathrm{H} 2$ and $\mathrm{H} 3$ of trans-2-pentenal). However, the conventional J-res experiments are not adequate for very closely resolved strong coupling cases; wherein, pure absorptive mode adiabatic z-filter type of J-res experiments may be considered. ${ }^{21}$

In summary, the known ideas of GIC based pure shift NMR strategy has been used, in order to overcome the resolution issues of 2D homonuclear NMR experiments (COSY and TOCSY) recorded on a tabletop $60 \mathrm{MHz}$ spectrometer. The proposed method has been demonstrated on different small organic molecules such as ibuprofen, trans-2-pentenal and a mixture of trans-2-pentenal and propanol. Herein, a significant amount of enhancement in the spectral resolution has been observed for the GIC processed spectra when compared with the conventional spectra recorded at $60 \mathrm{MHz}$. This type of experimental and processing approaches certainly helps in monitoring the structural changes that have taken place in the reaction analyte 
during chemical reaction processes, and thus could be of routine use in chemistry laboratories. We believe that a small modification in the NMR processing software (create a single macro by combining all the software patches) can make this process opaque to the normal user community and can become a routine feature on tabletop NMR spectrometers.

\section{Conflicts of interest}

There are no conflicts to declare.

\section{Acknowledgements}

The authors thank UM-DAE-CBS (University of MumbaiDepartment of Atomic Energy-Centre for Excellence in Basic Sciences) for supporting this work. KR (PDF/2016/002063) and VMRK (PDF/2016/000365) are thankful to DST-SERB (Department of Science and Technology-Science and Engineering Research Board), Govt. Of India, New Delhi for the funding and award of National Postdoctoral Fellowships.

\section{Notes and references}

1 (a) T. Niklas, D. Stalke and M. John, Chem. Commun., 2015, 51, 1275; (b) I. E. Ndukwe, A. Shchukina, K. Kazimierczuk and C. P. Butts, Chem. Commun., 2016, 52, 1276; (c) G. E. Wagner, P. Sakhaii, W. Bermel and K. Zangger, Chem. Commun., 2013, 49, 3155; (d) D. A. Foley, E. Bez, A. Codina, K. L. Colson, M. Fey, R. Krull, D. Piroli, M. T. Zell and B. L. Marquez, Anal. Chem., 2014, 86, 12008.

2 T. Parker, E. Limer, A. D. Watson, M. Defernez, D. Williamson and E. K. Kemsley, TrAC, Trends Anal. Chem., 2014, 57, 147.

3 A. Gerdova, M. Defernez, W. Jakes, E. Limer, C. McCallum, K. Nott, T. Parker, N. Rigby, A. Sagidullin, A. D. Watson, D. Williamson and E. K. Kemsley, Magnetic Resonance in Food Science: Defining Food by Magnetic Resonance, 2015, p. 17.

4 G. Pagès, A. Gerdova, D. Williamson, V. Gilard, R. Martino and M. Malet-Martino, Anal. Chem., 2014, 86, 11897.

5 W. Jakes, A. Gerdova, M. Defernez, A. D. Watson, C. McCallum, E. Limer, I. J. Colquhoun, D. C. Williamson and E. K. Kemsley, Food Chem., 2015, 175, 1.
6 E. Danieli, J. Mauler, J. Perlo, B. Blümich and E. Casanova, J. Magn. Reson., 2009, 198, 80.

7 V. Sans, L. Porwol, V. Dragone and L. Cronin, Chem. Sci., 2015, 6, 1258.

8 N. Zientek, C. Laurain, K. Meyer, M. Kraume, G. Guthausen and M. Maiwald, J. Magn. Reson., 2014, 249, 53.

9 S. K. Küster, E. Danieli, B. Blümich and F. Casanova, Phys. Chem. Chem. Phys., 2011, 13, 13172.

10 F. Dalitz, M. Cudaj, M. Maiwald and G. Guthausen, Prog. Nucl. Magn. Reson. Spectrosc., 2014, 60, 52.

11 (a) N. H. Meyer and K. Zangger, ChemPhysChem, 2014, 15, 49; (b) K. Zangger, Prog. Nucl. Magn. Reson. Spectrosc., 2015, 8687, 1; (c) L. Castañar and T. Parella, Magn. Reson. Chem., 2015, 53, 399; (d) L. Castañar, Magn. Reson. Chem., 2017, 55, 47; (e) R. W. Adams, eMagRes, 2014, 3, 295.

12 (a) K. Zangger and H. Sterk, J. Magn. Reson., 1997, 124, 486; (b) N. H. Meyer and K. Zangger, Angew. Chem., Int. Ed., 2013, 52, 7143; (c) J. A. Aguilar, S. Faulkner, M. Nilsson and G. A. Morris, Angew. Chem., Int. Ed., 2010, 49, 3901.

13 (a) J. R. Garbow, D. P. Weitekamp and A. Pines, Chem. Phys. Lett., 1982, 93, 504; (b) A. Lupulescu, G. L. Olsen and L. Frydman, J. Magn. Reson., 2012, 218, 141; (c) J. A. Aguilar, M. Nilsson and G. A. Morris, Angew. Chem., Int. Ed., 2011, 50, 9716.

14 L. Castañar, P. Nolis, A. Virgili and T. Parella, Chem.-Eur. J., 2013, 19, 17283.

15 M. Foroozandeh, R. W. Adams, N. J. Meharry, D. Jeannerat, M. Nilsson and G. A. Morris, Angew. Chem., Int. Ed., 2014, 53, 6990.

16 (a) A. Fredi, P. Nolis, C. Cobas and T. Parella, J. Magn. Reson., 2016, 270, 161; (b) A. Fredi, P. Nolis, C. Cobas, G. E. Martin and T. Parella, J. Magn. Reson., 2016, 266, 16.

17 D. A. Snyder and R. Brüschweiler, J. Phys. Chem. A, 2009, 113, 12898.

18 G. E. Martin, R. T. Williamson, K. A. Blinov, C. G. Anklin and W. Bermel, Magn. Reson. Chem., 2012, 50, 691.

19 (a) J. A. Aguilar, A. A. Colbourne, J. Cassani, M. Nilsson and G. A. Morris, Angew. Chem., Int. Ed., 2012, 51, 6460; (b) M. Foroozandeh, R. W. Adams, M. Nilsson and G. A. Morris, J. Am. Chem. Soc., 2014, 136, 11867.

20 W. P. Aue, J. Karhan and R. R. Ernst, J. Chem. Phys., 1976, 64, 4226.

21 B. Luy, J. Magn. Reson., 2009, 201, 18. 